\title{
Interactions of Delta Shock Waves for Zero-Pressure Gas Dynamics with Energy Conservation Law
}

\author{
Wei Cai ${ }^{1}$ and Yanyan Zhang ${ }^{2}$ \\ ${ }^{1}$ The Basic Department, The First Aeronautic Institute of the Air Force, Xinyang 464000, China \\ ${ }^{2}$ College of Mathematics and Information Science, Xinyang Normal University, Xinyang 464000, China \\ Correspondence should be addressed to Yanyan Zhang; zyy@xynu.edu.cn
}

Received 1 April 2016; Accepted 15 May 2016

Academic Editor: Ming Mei

Copyright (c) 2016 W. Cai and Y. Zhang. This is an open access article distributed under the Creative Commons Attribution License, which permits unrestricted use, distribution, and reproduction in any medium, provided the original work is properly cited.

\begin{abstract}
We study the interactions of delta shock waves and vacuum states for the system of conservation laws of mass, momentum, and energy in zero-pressure gas dynamics. The Riemann problems with initial data of three piecewise constant states are solved case by case, and four different configurations of Riemann solutions are constructed. Furthermore, the numerical simulations completely coinciding with theoretical analysis are shown.
\end{abstract}

\section{Introduction}

As is well known, the system of zero-pressure gas dynamics consisting of conservation laws of mass and momentum, which is also called the transport equations, or Euler equations for pressureless fluids, has been extensively investigated since the 90 s of 20th century. It is derived from Boltzmann equations [1] and the flux-splitting scheme of the full compressible Euler equations $[2,3]$ and can be used to describe the motion process of free particles sticking together under collision [4] and the formation of large-scale structures in the universe $[5,6]$.

However, we have to mention that, as having no pressure, the energy transport must be taken into account for the considered media. Therefore, it is very necessary to consider the conservation law of energy in zero-pressure gas dynamics. To this end, we study the one-dimensional zero-pressure gas dynamics governed by the conservation laws of mass, momentum, and energy:

$$
\begin{aligned}
\rho_{t}+(\rho u)_{x} & =0, \\
(\rho u)_{t}+\left(\rho u^{2}\right)_{x} & =0, \\
\left(\frac{\rho u^{2}}{2}+H\right)_{t}+\left(\left(\frac{\rho u^{2}}{2}+H\right) u\right)_{x} & =0,
\end{aligned}
$$

where $\rho$ and $u$ represent the density and velocity, respectively, $H=\rho \tau$ is the internal energy and assumed to be nonnegative, and $\tau$ is the internal energy per unit mass. The regions in the physical space where $\rho=0$ and $H=0$ are identified with the vacuum regions of the flow. Here, $H$ is considered as an independent variable just for convenience.

System (1) was early studied by Kraiko [7]. In contrast to the traditional zero-pressure gas dynamics system that contains only the conservation laws of mass and momentum, in order to construct the solution of (1) for arbitrary initial data, a new type of discontinuities which are different from the classical ones and carry mass, impulse, and energy are needed. In $[8,9]$, system (1) was further discussed. Some special integral identities were introduced to define the delta-shock solutions and construct the Rankine-Hugoniot relation for delta shock waves. Moreover, using these integral identities, the balance laws describing mass, momentum, and energy transport from the area outside the delta shock wave front onto its front were derived. What is more, the delta shock wave type solutions for multidimensional zeropressure gas dynamics with the energy conservation law were defined in [10].

A delta shock wave is a generalization of an ordinary shock wave. Roughly speaking, it is a kind of discontinuity, on which at least one of the state variables may develop 
an extreme concentration in the form of a weighted Dirac delta function with the discontinuity as its support. It is more compressive than an ordinary shock wave and more characteristics enter the discontinuity line. Physically, the delta shock waves describe the process of formation of the galaxies in the universe and the process of concentration of particles. As for delta shock waves, there are numerous excellent papers, see [11-21] and so forth. Nevertheless, compared to these results, a distinctive feature for (1) is that the Dirac delta functions develop in both state variables $\rho$ and $H$ simultaneously, which is quite different from those aforementioned, in which only one state variable contains the Dirac delta function. In fact, the theory of delta shock waves with Dirac delta functions developing in both state variables has been established by Yang and Zhang [22, 23] for a class of $2 \times 2$ nonstrictly hyperbolic systems of conservation laws.

In the past over two decades, the investigation of interactions of delta shock waves has been increasingly active. This is important not only because of their significance in practical applications but also because of their basic role as building blocks for the general mathematical theory of quasilinear hyperbolic equations. And the results on interactions are also touchstones for the numerical schemes. Specifically, Sheng and Zhang [18] discussed the overtaking of delta shock waves and vacuum states in one-dimensional zero-pressure gas dynamics. By solving the two-dimensional Riemann problems for zero-pressure gas dynamics with three constant states, Cheng et al. [24] studied the interactions among delta-shock waves, vacuums, and contact discontinuities. In addition, with the help of a generalized plane wave solution, Yang [25] studied a type of generalized plane delta-shock wave for the $n$-dimensional zero-pressure gas dynamics and investigated the overtaking of two plane delta shocks. For more works on the interactions of delta shock waves, we refer to [26-29] and so forth.

Motivated by the discussions above, in the present paper, we are concerned with the interactions among delta shock waves, vacuum states, and contact discontinuities in solutions. Therefore, we study the Riemann problem of (1) with initial data of three piecewise constant states as follows:

$$
(\rho, u, H)(0, x)= \begin{cases}\left(\rho_{-}, u_{-}, H_{-}\right), & x<x_{01}, \\ \left(\rho_{m}, u_{m}, H_{m}\right), & x_{01}<x<x_{02}, \\ \left(\rho_{+}, u_{+}, H_{+}\right), & x>x_{02},\end{cases}
$$

where $\rho_{i}, u_{i}, H_{i}(i= \pm, m)$ are arbitrary constants and $x_{01}, x_{02}$ are any two fixed points on $x$-axis.

We will deal with the Riemann problem (1), (2) case by case along with constructing the solutions. For this purpose, it is necessary to consider whether two adjacent waves intersect and interact with each other when constructing the global solution. However, it is often not so easy to see whether two delta shock waves meet and how they interact with each other. Therefore, some technical treatments are needed.

This paper is arranged as follows. In Section 2, the delta shock solution of (1) is reviewed and a general case when the delta shock wave is emitted at the beginning with a nonzero initial data is considered. Section 3 discusses the interactions of the delta shock waves and vacuum states. The Riemann solutions of (1), (2) are constructed globally. Finally, four kinds of numerical simulations coinciding with the theoretical analysis are presented in Section 4.

\section{Delta-Shock Solution}

This section briefly reviews the delta shock solution of (1) with the initial data

$$
(\rho, u, H)(0, x)=\left(\rho_{ \pm}, u_{ \pm}, H_{ \pm}\right), \quad \pm x>0,
$$

and the detailed study can be found in [30].

System (1) has a triple eigenvalue $\lambda=u$ and two right eigenvectors $\overrightarrow{r_{1}}=(1,0,0)^{T}$ and $\overrightarrow{r_{2}}=(0,0,1)^{T}$. Since $\nabla \lambda \cdot \overrightarrow{r_{i}} \equiv$ 0 for $i=1,2, \lambda$ is linearly degenerate, which means that the elementary waves involve only contact discontinuities. The self-similar solution $(\rho, v, H)(\xi)(\xi=x / t)$ is constructed by two cases.

For the case $u_{-} \leq u_{+}$, the solution containing two contact discontinuities and a vacuum state besides two constants is expressed as

$$
(\rho, u, H)(\xi)= \begin{cases}\left(\rho_{-}, u_{-}, H_{-}\right), & -\infty<\xi<u_{-} \\ (0, u(\xi), 0), & u_{-} \leq \xi \leq u_{+} \\ \left(\rho_{+}, u_{+}, H_{+}\right), & u_{+}<\xi<+\infty\end{cases}
$$

where $u(\xi)$ is a smooth function satisfying $u\left(u_{-}\right)=u_{-}$and $u\left(u_{+}\right)=u_{+}$.

For the case $u_{-}>u_{+}$, the singularity of solutions must develop because of the overlap of characteristic lines. Therefore, the solution involving a delta shock wave is introduced. form

Let $\left(\rho, u, H ; w, u_{\delta}, h\right)$ be the delta shock solution of the

$$
\begin{aligned}
& (\rho, u, H)(t, x) \\
& = \begin{cases}\left(\rho_{-}, u_{-}, H_{-}\right)(t, x), & x<x(t), \\
\left(w(t) \delta(x-x(t)), u_{\delta}(t), h(t) \delta(x-x(t))\right), & x=x(t), \\
\left(\rho_{+}, u_{+}, H_{+}\right)(t, x), & x>x(t),\end{cases}
\end{aligned}
$$

and then the following generalized Rankine-Hugoniot relation holds

$$
\begin{aligned}
\frac{\mathrm{d} x}{\mathrm{~d} t} & =u_{\delta}, \\
\frac{\mathrm{d} w}{\mathrm{~d} t} & =-[\rho] u_{\delta}+[\rho u], \\
\frac{\mathrm{d}\left(w u_{\delta}\right)}{\mathrm{d} t} & =-[\rho u] u_{\delta}+\left[\rho u^{2}\right],
\end{aligned}
$$




$$
\begin{aligned}
\frac{\mathrm{d}\left(w u_{\delta}^{2} / 2+h\right)}{\mathrm{d} t}= & -\left[\frac{\rho u^{2}}{2}+H\right] u_{\delta} \\
& +\left[\left(\frac{\rho u^{2}}{2}+H\right) u\right],
\end{aligned}
$$

where $[\rho]=\rho_{-}-\rho_{+}$. In order to ensure the uniqueness, the delta shock wave should satisfy the entropy condition

$$
u_{+}<u_{\delta}<u_{-}
$$

which means that the characteristics on both sides of the discontinuity are in-coming.

Under the entropy condition (7), by solving the ordinary differential equations (6) with the initial data $t=0: x(0)=$ $0, w(0)=0, h(0)=0, u_{\delta}(0)=0$, one has

$$
\begin{aligned}
& x(t)=\frac{\sqrt{\rho_{-}} u_{-}+\sqrt{\rho_{+}} u_{+}}{\sqrt{\rho_{-}}+\sqrt{\rho_{+}}} t, \\
& w(t)=\sqrt{\overline{\rho_{-} \rho_{+}}}\left(u_{-}-u_{+}\right) t, \\
& u_{\delta}(t)=\frac{\sqrt{\rho_{-}} u_{-}+\sqrt{\rho_{+}} u_{+}}{\sqrt{\rho_{-}}+\sqrt{\rho_{+}}},
\end{aligned}
$$

$h(t)$

$$
\begin{aligned}
& =\frac{\rho_{-} \rho_{+}\left(u_{-}-u_{+}\right)^{2}+2\left(\sqrt{\rho_{-}}+\sqrt{\rho_{+}}\right)\left(H_{-} \sqrt{\rho_{+}}+H_{+} \sqrt{\rho_{-}}\right)}{2\left(\sqrt{\rho_{-}}+\sqrt{\rho_{+}}\right)^{2}}\left(u_{-}\right. \\
& \left.-u_{+}\right) t .
\end{aligned}
$$

For convenience, we now consider a special case when a delta shock wave is emitted at the beginning with the initial data

$$
\begin{aligned}
t & =0: \\
x(0) & =0, \\
w(0) & =m_{0}, \\
h(0) & =h_{0}, \\
u_{\delta}(0) & =u_{0}
\end{aligned}
$$

satisfying $u_{-}>u_{0}>u_{+}$. It yields from (6) and (9) that

$$
x(t)= \begin{cases}\frac{m_{0} u_{0} t+\left[\rho u^{2}\right] t^{2} / 2}{m_{0}+[\rho u] t}, & \rho_{-}=\rho_{+}, \\ \frac{[\rho u] t+m_{0}-w(t)}{[\rho]}, & \rho_{-} \neq \rho_{+},\end{cases}
$$

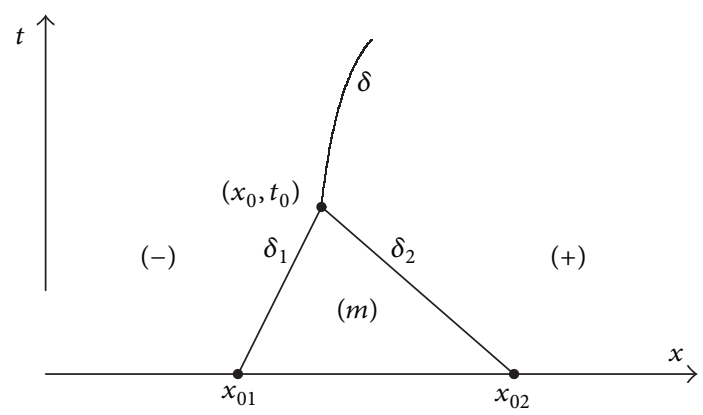

FIGURE 1: $u_{-}>u_{m}>u_{+}$.

$$
\begin{aligned}
& w(t)=\left(m_{0}^{2}+2 m_{0}\left([\rho u]-[\rho] u_{0}\right) t\right. \\
& \left.+\rho_{-} \rho_{+}\left(u_{-}-u_{+}\right)^{2} t^{2}\right)^{1 / 2} \\
& u_{\delta}(t)=\frac{m_{0} u_{0}+\left[\rho u^{2}\right] t-[\rho u] x}{w(t)}, \\
& h(t)=-\left[\frac{\rho u^{2}}{2}+H\right] x+\left[\left(\frac{\rho u^{2}}{2}+H\right) u\right] t+\frac{m_{0} u_{0}^{2}}{2} \\
& +h_{0}-\frac{w(t) u_{\delta}^{2}}{2} .
\end{aligned}
$$

One can check that the delta shock solution (10) satisfies the following:

(1) $x^{\prime}(t)$ is a monotone function of $t$.

(2) If $\rho_{-}+\rho_{+}>0$, then

$$
\lim _{t \rightarrow+\infty} u_{\delta}(t)=\frac{\sqrt{\rho_{-}} u_{-}+\sqrt{\rho_{+}} u_{+}}{\sqrt{\rho_{-}}+\sqrt{\rho_{+}}} .
$$

While if $\rho_{-}=\rho_{+}=0$, then $u_{\delta}=u_{0}$.

(3) $u_{+}<u_{\delta}<u_{-}$.

\section{Interactions of Delta Shock Waves}

In this section, we analyze the interactions of delta shock waves. To ensure that all the cases are covered completely, according to the relation among $u_{-}, u_{m}, u_{+}$, our discussion is divided into four cases:
(1) $u_{-}>u_{m}>u_{+}$;
(2) $u_{-}>u_{+}>u_{m}$;
(3) $u_{+}>u_{-}>u_{m}$;
(4) $u_{+}>u_{m}>u_{-}$.

Case $1\left(u_{-}>u_{m}>u_{+}\right)$. In this case, two delta shock waves $\delta_{1}$ and $\delta_{2}$ will be emitted from $\left(x_{01}, 0\right)$ and $\left(x_{02}, 0\right)$, respectively, as shown in Figure 1.

According to what has been discussed in Section 2, these two delta shock waves are uniquely determined by 


$$
\delta_{1}:\left\{\begin{array}{l}
x_{1}(t)=\frac{\sqrt{\rho_{-}} u_{-}+\sqrt{\rho_{m}} u_{m}}{\sqrt{\rho_{-}}+\sqrt{\rho_{m}}} t+x_{01}, \\
u_{\delta_{1}}=\frac{\sqrt{\rho_{-}} u_{-}+\sqrt{\rho_{m}} u_{m}}{\sqrt{\rho_{-}}+\sqrt{\rho_{m}}}, \\
w_{\delta_{1}}(t)=\sqrt{\rho_{-} \rho_{m}}\left(u_{-}-u_{m}\right) t, \\
h_{\delta_{1}}(t)=\frac{\rho_{-} \rho_{m}\left(u_{-}-u_{m}\right)^{2}+2\left(\sqrt{\rho_{-}}+\sqrt{\rho_{m}}\right)\left(H_{-} \sqrt{\rho_{m}}+H_{m} \sqrt{\rho_{-}}\right)}{2\left(\sqrt{\rho_{-}}+\sqrt{\rho_{m}}\right)^{2}}\left(u_{-}-u_{m}\right) t, \\
\delta_{\delta_{2}}=\frac{\sqrt{\rho_{m}} u_{m}+\sqrt{\rho_{+}} u_{+}}{\sqrt{\rho_{m}}+\sqrt{\rho_{+}}}, \quad \begin{array}{l}
x_{2}(t)=\frac{\sqrt{\rho_{m}} u_{m}+\sqrt{\rho_{+}} u_{+}}{\sqrt{\rho_{+}}+\sqrt{\rho_{m}}} t+x_{02}, \\
w_{\delta_{2}}(t)=\sqrt{\rho_{m} \rho_{+}}\left(u_{m}-u_{+}\right) t, \\
h_{\delta_{2}}(t)=\frac{\rho_{m} \rho_{+}\left(u_{m}-u_{+}\right)^{2}+2\left(\sqrt{\rho_{m}}+\sqrt{\rho_{+}}\right)\left(H_{m} \sqrt{\rho_{+}}+H_{+} \sqrt{\rho_{m}}\right)}{2\left(\sqrt{\rho_{m}}+\sqrt{\rho_{+}}\right)^{2}}\left(u_{m}-u_{+}\right) t .
\end{array}
\end{array}\right.
$$

We have $u_{+}<u_{\delta_{2}}<u_{m}<u_{\delta_{1}}<u_{-}$by entropy condition (6), which means that $\delta_{1}$ will overtake $\delta_{2}$ at a finite time. The intersection point $\left(x_{0}, t_{0}\right)$ is calculated by

$$
\begin{aligned}
& x_{0}-x_{01}=u_{\delta_{1}} t_{0}, \\
& x_{0}-x_{02}=u_{\delta_{2}} t_{0},
\end{aligned}
$$

which yields that

$$
\left(x_{0}, t_{0}\right)=\left(\frac{x_{02}-x_{01}}{u_{\delta_{1}}-u_{\delta_{2}}} u_{\delta_{1}}+x_{01}, \frac{x_{02}-x_{01}}{u_{\delta_{1}}-u_{\delta_{2}}}\right) .
$$

At the intersection $\left(x_{0}, t_{0}\right)$, the new initial data are formed as follows:

$$
t=t_{0}:\left\{\begin{array}{l}
x\left(t_{0}\right)=x_{1}\left(t_{0}\right)=x_{2}\left(t_{0}\right), \\
w\left(t_{0}\right)=w_{\delta_{1}}\left(t_{0}\right)+w_{\delta_{2}}\left(t_{0}\right):=w_{0}, \\
h\left(t_{0}\right)=h_{\delta_{1}}\left(t_{0}\right)+h_{\delta_{2}}\left(t_{0}\right):=h_{0}, \\
u_{\delta}\left(t_{0}\right)=\frac{w_{\delta_{1}}\left(t_{0}\right) u_{\delta_{1}}+w_{\delta_{2}}\left(t_{0}\right) u_{\delta_{2}}}{w_{\delta_{1}}\left(t_{0}\right)+w_{\delta_{2}}\left(t_{0}\right)}:=u_{\delta}^{0}
\end{array}\right.
$$

satisfying $u_{\delta_{1}}>u_{\delta}^{0}>u_{\delta_{2}}$.

In view of $u_{-}>u_{+}$, a new delta shock wave will generate after interaction and we denote it with $\delta: x=x(t)$. The trajectory, velocity, and weights $\left(x(t), u_{\delta}(t), w(t), h(t)\right)$ of $\delta$ can be uniquely obtained by solving the ordinary differential equations (6) with the initial date (16). The detail is omitted.

Thus, the result of interaction of two delta shock waves is still a single delta shock wave. This fact can be formulated as

$$
\delta+\delta \longrightarrow \delta
$$

Case $2\left(u_{-}>u_{+}>u_{m}\right.$ (when $u_{m}>u_{+}>u_{-}$, the structure of solution is similar)). In this situation, a delta shock wave $\delta_{1}$ determined by (12) is emitted from $\left(x_{01}, 0\right)$ and two contact discontinuities $J_{1}: u=u_{m}$ and $J_{2}: u=u_{+}$with a vacuum in between are emitted from $\left(x_{02}, 0\right)$, as shown in Figure 2 .

Since the propagating speed of $\delta_{1}$ satisfies $u_{m}<u_{\delta_{1}}<u_{-}$, so $\delta_{1}$ must meet the contact discontinuity $J_{1}: u=u_{m}$ at $t_{1}=$ $\left(x_{02}-x_{01}\right) /\left(u_{\delta_{1}}-u_{m}\right)$, and a new delta shock wave $\delta_{2}: x=$ $x_{2}(t)$ forms, which is subjected to the generalized RankineHugoniot relation

$$
\begin{aligned}
& \frac{\mathrm{d} x}{\mathrm{~d} t}=u_{\delta}, \\
& \frac{\mathrm{d} w}{\mathrm{~d} t}=\rho_{-} u_{-}-\rho_{-} u_{\delta}, \\
& \frac{\mathrm{d}\left(w u_{\delta}\right)}{\mathrm{d} t}=\rho_{-} u_{-}^{2}-\rho_{-} u_{-} u_{\delta}, \\
& \frac{\mathrm{d}}{\mathrm{d} t}\left(\frac{w u_{\delta}^{2}}{2}+h\right)=\left(\frac{\rho_{-} u_{-}^{2}}{2}+H_{-}\right) u_{-} \\
&-\left(\frac{\rho_{-} u_{-}^{2}}{2}+H_{-}\right) u_{\delta}
\end{aligned}
$$

with the initial data

$$
t=t_{1}:\left\{\begin{array}{l}
x\left(t_{1}\right)=x_{1}\left(t_{1}\right):=x_{1}, \\
w\left(t_{1}\right)=w_{\delta_{1}}\left(t_{1}\right):=w_{1}, \\
h\left(t_{1}\right)=h_{\delta_{1}}\left(t_{1}\right):=h_{1}, \\
u_{\delta}\left(t_{1}\right)=u_{\delta_{1}}\left(t_{1}\right):=u_{\delta_{1}} .
\end{array}\right.
$$




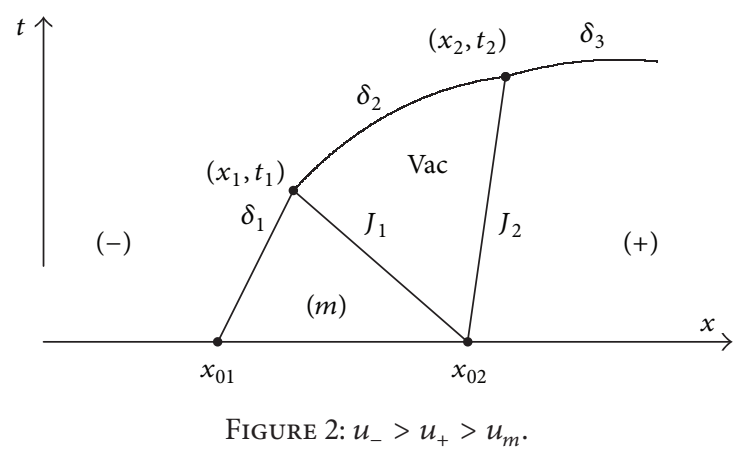

Therefore, by solving (18) and (19), we have

$$
\begin{aligned}
& x_{2}(t)=\frac{1}{\rho_{-}}\left(w_{1}+\rho_{-} u_{-}\left(t-t_{1}\right)\right. \\
&\left.-\left(w_{1}^{2}+2 w_{1} \rho_{-}\left(u_{-}-u_{\delta_{1}}\right)\left(t-t_{1}\right)\right)^{1 / 2}\right)+x_{1}, \\
& w_{2}(t)=\left(w_{1}^{2}+2 w_{1} \rho_{-}\left(u_{-}-u_{\delta_{1}}\right)\left(t-t_{1}\right)\right)^{1 / 2}, \\
& u_{\delta_{2}}(t)=u_{-}-\frac{w_{1}\left(u_{-}-u_{\delta_{1}}\right)}{w_{2}(t)}, \\
& h_{2}(t)=\left(\frac{\rho_{-} u_{-}^{2}}{2}+H_{-}\right) u_{-}\left(t-t_{1}\right)-\left(\frac{\rho_{-} u_{-}^{2}}{2}+H_{-}\right) \\
& \cdot\left(x_{2}(t)-x_{1}\right)+\frac{w_{1} u_{\delta_{1}}^{2}}{2}+h_{1}-\frac{w_{2}(t) u_{\delta_{2}}^{2}(t)}{2} .
\end{aligned}
$$

It is clear that $\delta_{2}$ will cross the vacuum region with a varying propagation speed. Noting that $\lim _{t \rightarrow+\infty} u_{\delta_{2}}(t)=$ $u_{-}>u_{+}$, so $\delta_{2}$ will penetrate over the whole vacuum region and then meet $J_{2}: u=u_{+}$at a finite time. The intersection $\left(x_{2}, t_{2}\right)$ is determined by

$$
\begin{aligned}
x_{2} & =\frac{1}{\rho_{-}}\left(w_{1}+\rho_{-} u_{-}\left(t_{2}-t_{1}\right)\right. \\
& \left.-\left(w_{1}^{2}+2 w_{1} \rho_{-}\left(u_{-}-u_{\delta_{1}}\right)\left(t_{2}-t_{1}\right)\right)^{1 / 2}\right)+x_{1}, \\
x_{2} & =u_{+} t_{2}+x_{02} .
\end{aligned}
$$

At $\left(x_{2}, t_{2}\right)$, a new initial value problem is formed and can be solved similar to Case 1 . We denote the delta shock wave connecting two constant states $\left(\rho_{-}, u_{-}, H_{-}\right)$and $\left(\rho_{+}, u_{+}, H_{+}\right)$ with $\delta_{3}$ after the interaction of $\delta_{2}$ and $J_{2}$.

The conclusion of this case is that the delta shock wave will penetrate over the whole vacuum region between two contact discontinuities. This fact is expressed as

$$
\delta+J+\mathrm{Vac}+J \longrightarrow \delta .
$$

Case $3\left(u_{+}>u_{-}>u_{m}\right.$ (when $u_{m}>u_{-}>u_{+}$, the structure of solution is similar)). Similar to Case 2 , there are a delta shock wave, two contact discontinuities, and a vacuum near $t=0$ on the $(x, t)$-plane, as shown in Figure 3.

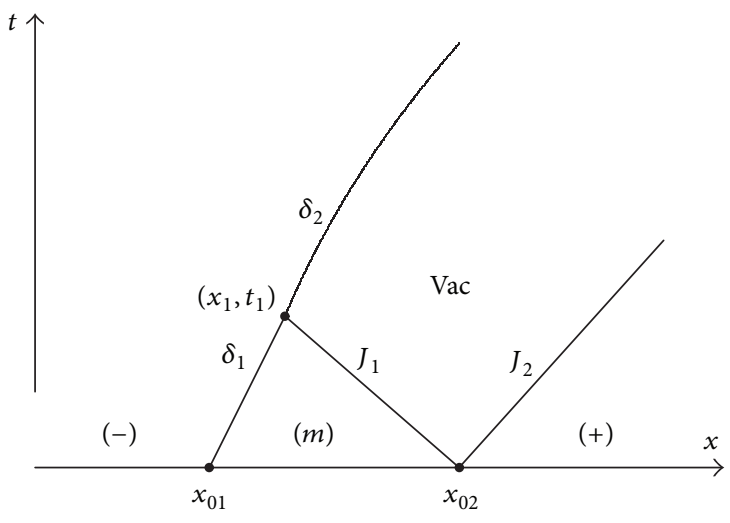

FIGURE 3: $u_{+}>u_{-}>u_{m}$.

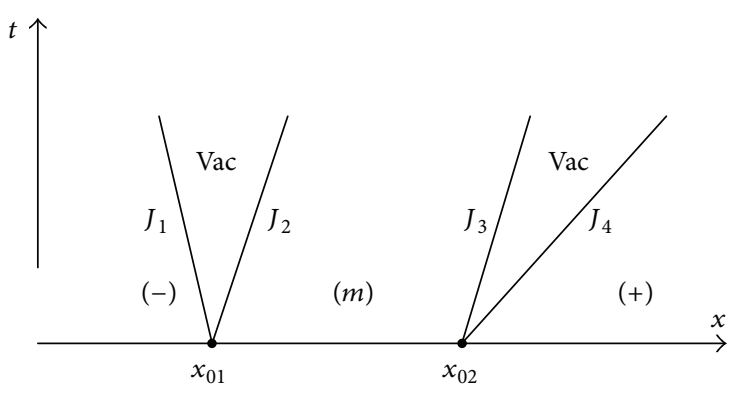

FIGURE 4: $u_{+}>u_{m}>u_{-}$.

The delta shock wave $\delta_{1}$ collides with $J_{1}$ at first and a new delta shock wave $\delta_{2}$ generates. However, since $\lim _{t \rightarrow+\infty} u_{\delta_{2}}(t)=u_{-}<u_{+}, \delta_{2}$ cannot penetrate over the vacuum region and finally has $x_{2}(t)=u_{-} t+x_{02}$ as its asymptote. This fact is symbolized as

$$
\delta+J+\mathrm{Vac}+J \longrightarrow \delta+\mathrm{Vac}+J .
$$

Case $4\left(u_{+}>u_{m}>u_{-}\right)$. In this situation, both the contact discontinuities with a vacuum state in between are emitted from $\left(x_{01}, 0\right)$ and $\left(x_{02}, 0\right)$, respectively. Noting that the contact discontinuities $J_{2}$ and $J_{3}$ own the same propagating speed, thus there is no collision of waves and the solution is expressed as

$$
\begin{aligned}
& (\rho, u, H)(t, x) \\
& \quad= \begin{cases}\left(\rho_{-}, u_{-}, H_{-}\right), & x<u_{-} t+x_{01}, \\
\operatorname{Vac}, & u_{-} t+x_{01}<x<u_{m} t+x_{01}, \\
\left(\rho_{m}, u_{m}, H_{m}\right), & u_{m} t+x_{01}<x<u_{m} t+x_{02}, \\
\operatorname{Vac}, & u_{m} t+x_{02}<x<u_{+} t+x_{02}, \\
\left(\rho_{+}, u_{+}, H_{+}\right), & x>u_{+} t+x_{02},\end{cases}
\end{aligned}
$$

which is called a collisionless solution, as shown in Figure 4. 


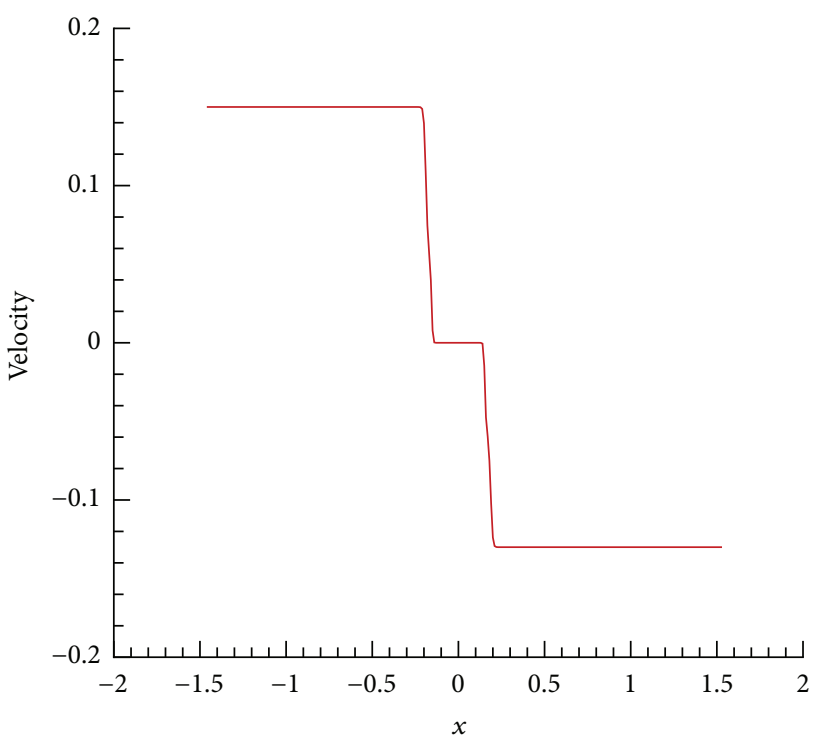

(a)

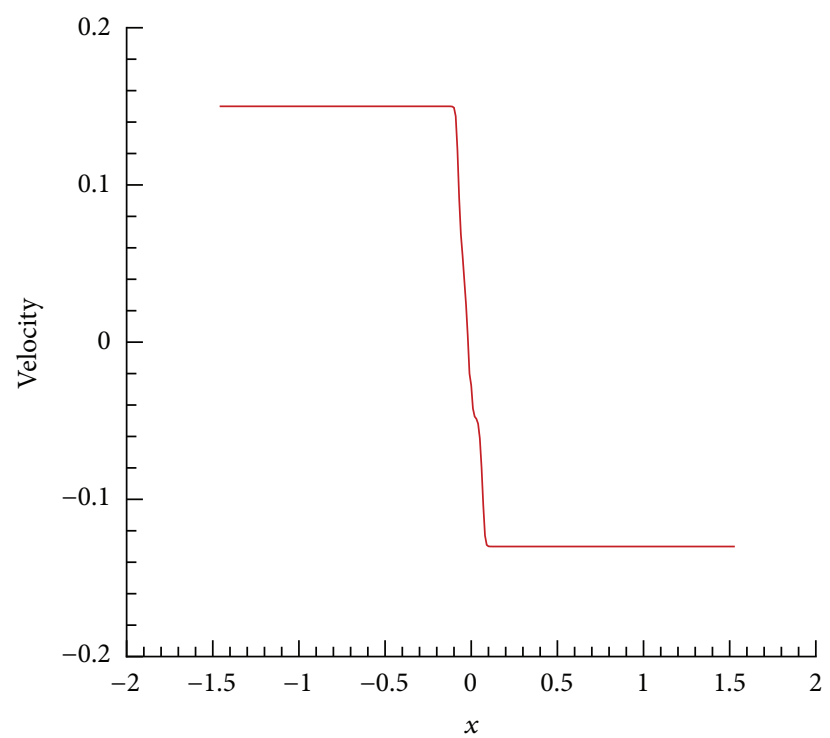

(b)

Figure 5: Numerical results of $u$ at $t=1$ (a) and $t=6.5$ (b) for Data 1 .

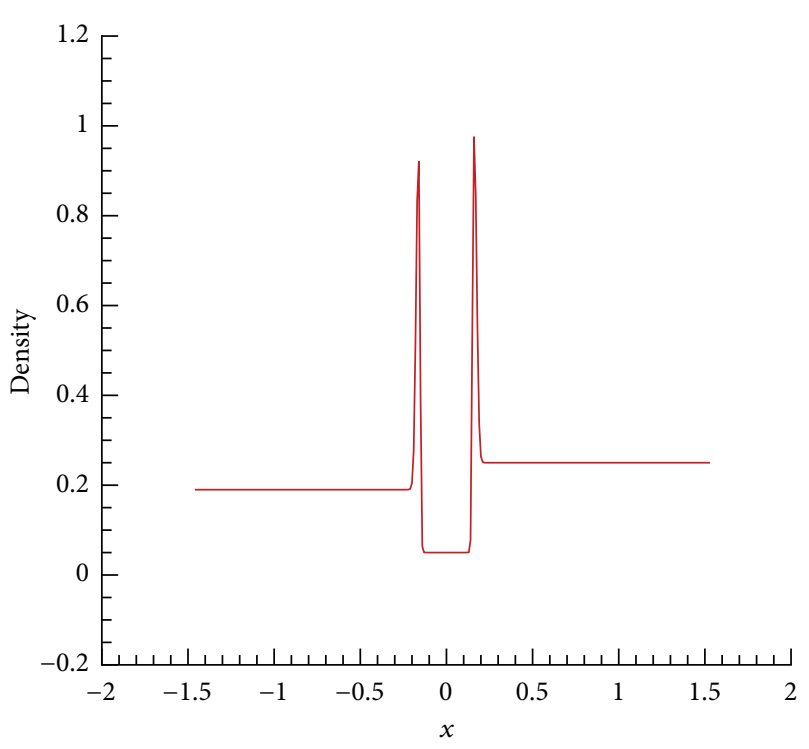

(a)

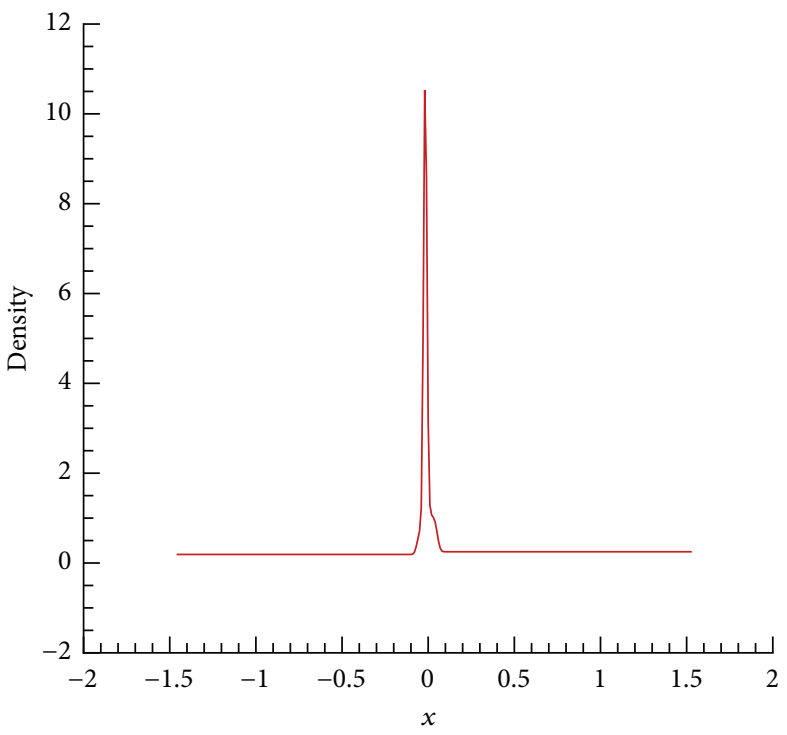

(b)

Figure 6: Numerical results of $\rho$ at $t=1$ (a) and $t=6.5$ (b) for Data 1 .

\section{Numerical Simulations}

In order to verify the validity of the interactions of delta shock waves and vacuum states mentioned in Section 3, we present some representative numerical simulations in this section. Many more numerical tests have been performed to make sure that what are presented are not numerical artifacts.

To discretize the system, we employ the second-order nonoscillatory central schemes [31] with $300 \times 300$ cells and $\mathrm{CFL}=0.475$. In what follows, by taking $x_{01}=-0.2$ and $x_{02}=$ 0.2 , we simulate the interaction of waves by four cases. For convenience, each situation will be simulated at two different times.

Case $1\left(u_{-}>u_{m}>u_{+}\right)$. We take the initial data as follows:

Data 1: $(\rho, u, H)(0, x)$

$$
= \begin{cases}(0.19,0.15,0.50), & x<-0.2, \\ (0.05,0.00,0.30), & -0.2<x<0.2, \\ (0.25,-0.13,0.01), & x>0.2 .\end{cases}
$$

The numerical results are presented by Figures 5-7. 


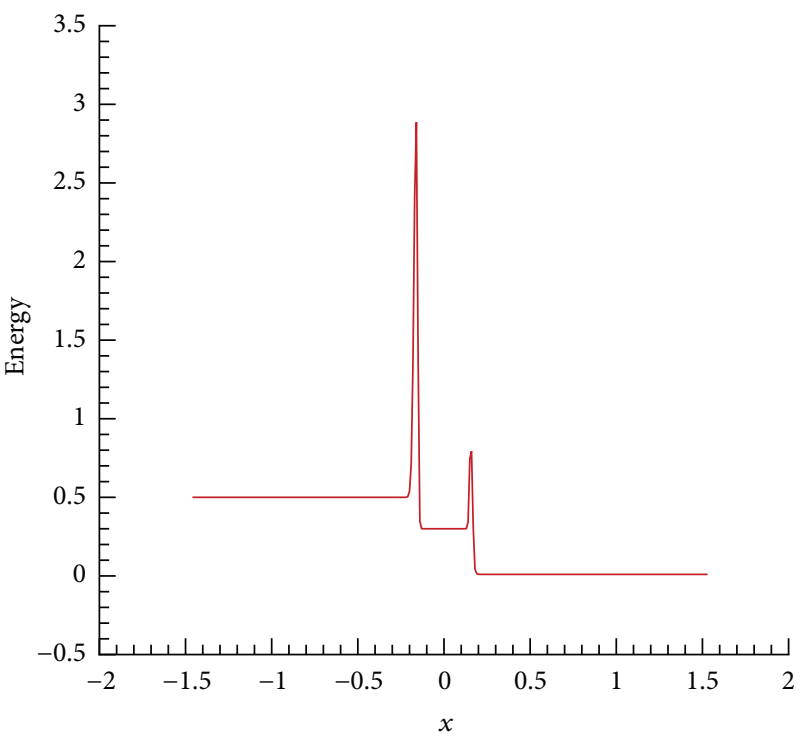

(a)

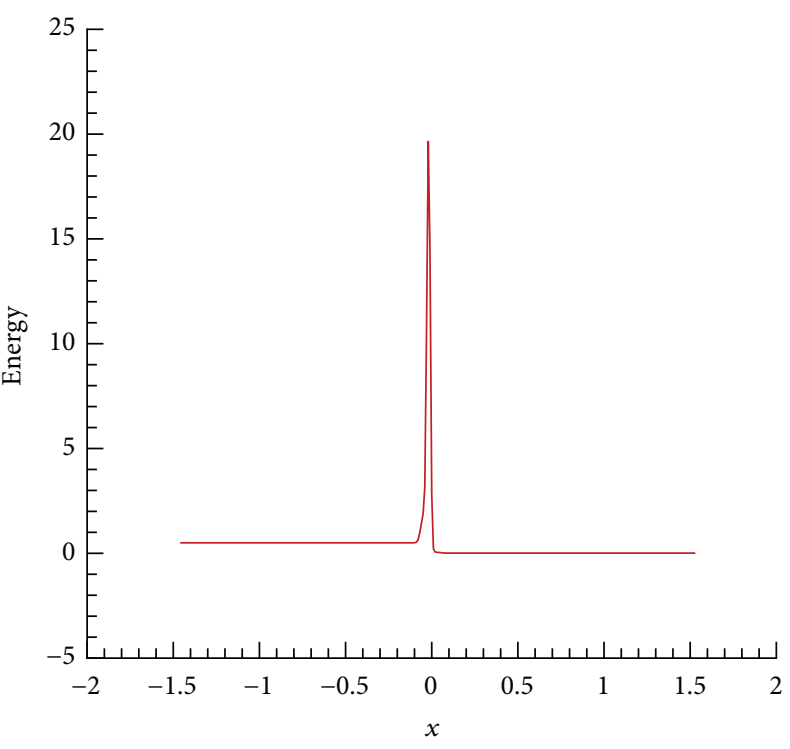

(b)

Figure 7: Numerical results of $H$ at $t=1$ (a) and $t=6.5$ (b) for Data 1.

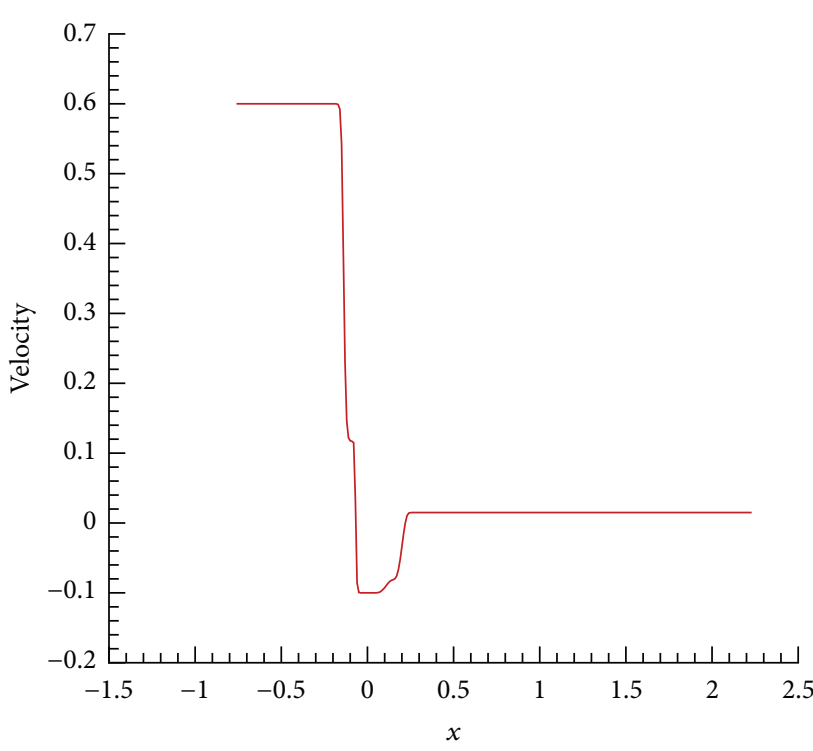

(a)

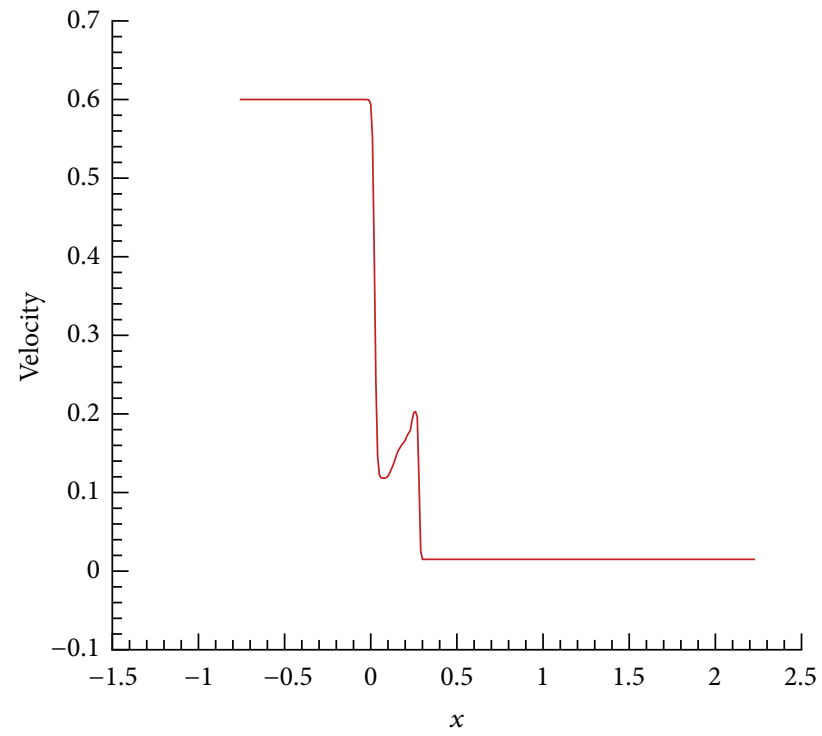

(b)

Figure 8: Numerical results of $u$ at $t=0.8$ (a) and $t=2.9$ (b) for Data 2.

We observe from Figures 5-7 that when $t=1$, two delta shock waves appear at $(-0.2,0)$ and $(0.2,0)$, respectively. As $t$ increases, they will overtake each other and finally unify into a new delta shock wave at $t=6.5$.

Case $2\left(u_{-}>u_{+}>u_{m}\right)$. We choose the following initial data

Data 2: $(\rho, u, H)(0, x)$

$$
= \begin{cases}(0.20,0.60,0.16), & x<-0.2, \\ (0.30,-0.10,0.30), & -0.2<x<0.2, \\ (0.25,0.015,0.20), & x>0.2 .\end{cases}
$$

From Figures 8-10, we can clearly see that, at $t=0.8$, a delta shock wave and two contact discontinuities with a vacuum state in between are emitted from $(-0.2,0)$ and $(0.2,0)$, respectively. However, at $t=2.9$, the delta shock wave penetrates over the whole vacuum region, and a new delta shock wave generates.

Case $3\left(u_{+}>u_{-}>u_{m}\right)$. The initial data are

Data 3: $(\rho, u, H)(0, x)$

$$
= \begin{cases}(0.36,0.18,0.60), & x<-0.2, \\ (0.30,-0.05,0.30), & -0.2<x<0.2, \\ (0.20,0.40,0.70), & x>0.2 .\end{cases}
$$

The numerical results are shown in Figures 11-13. 


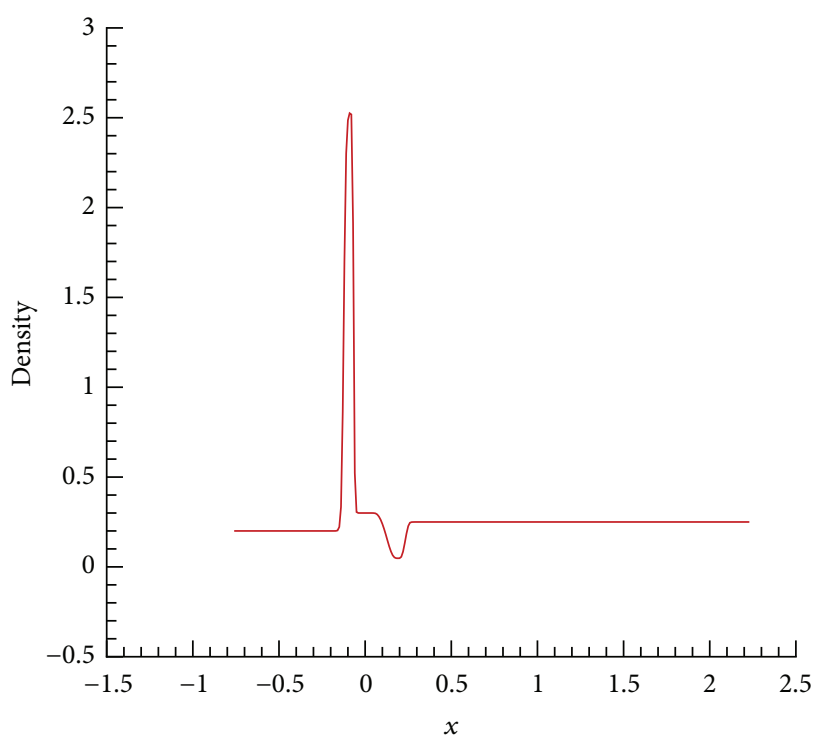

(a)

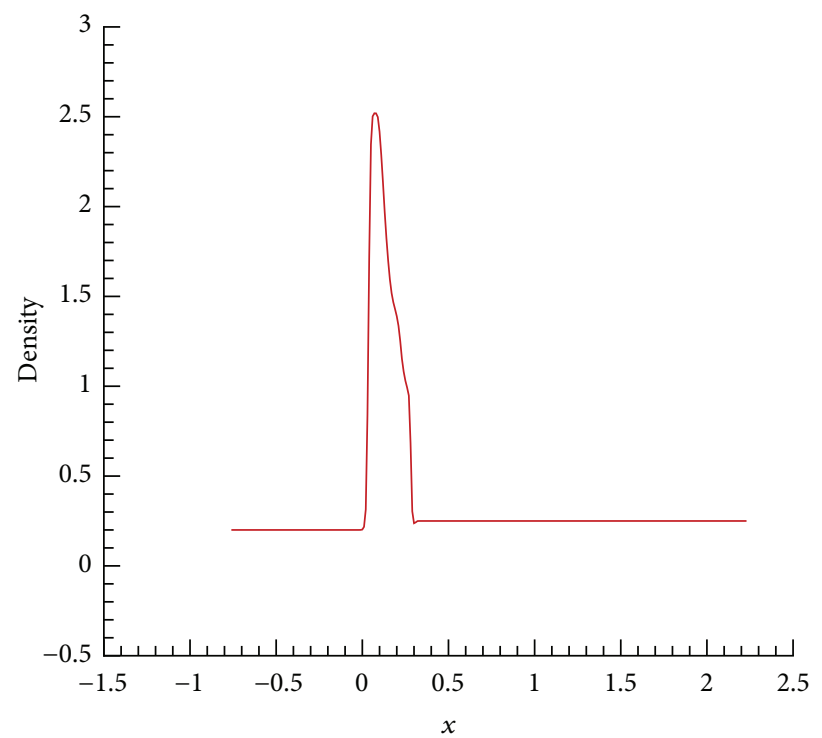

(b)

FIGURE 9: Numerical results of $\rho$ at $t=0.8$ (a) and $t=2.9$ (b) for Data 2.

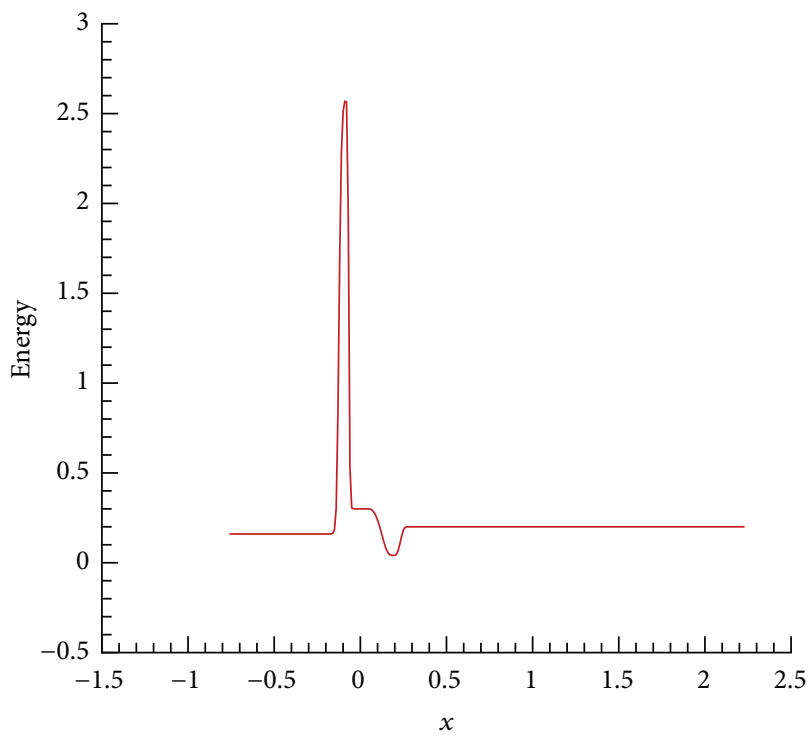

(a)

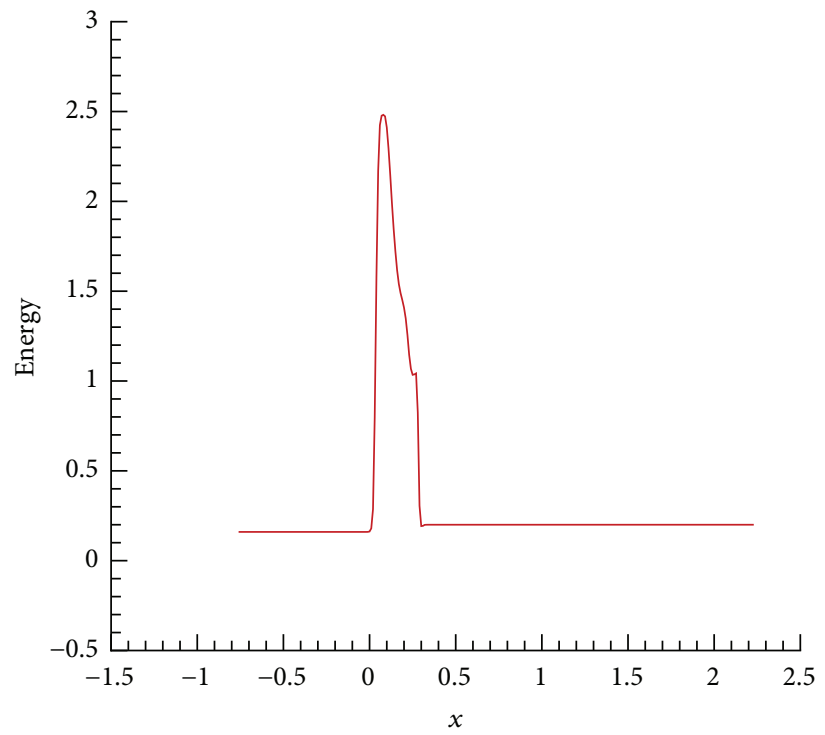

(b)

Figure 10: Numerical results of $H$ at $t=0.8$ (a) and $t=2.9$ (b) for Data 2.

Figures 11-13 imply that a delta shock wave is emitted from $(-0.2,0)$, and two contact discontinuities with a vacuum in between are emitted from $(0.2,0)$ at $t=0.5$. But the delta shock wave can not penetrate over the whole vacuum region even though time is on the increase. In this process, the region of vacuum state keeps expanding.
Case $4\left(u_{+}>u_{m}>u_{-}\right)$. We select the initial data to be

Data 4: $(\rho, u, H)(0, x)$

$$
= \begin{cases}(1.05,-0.10,0.70), & x<-0.2, \\ (0.70,0.00,0.30), & -0.2<x<0.2, \\ (1.06,0.10,0.90), & x>0.2 .\end{cases}
$$




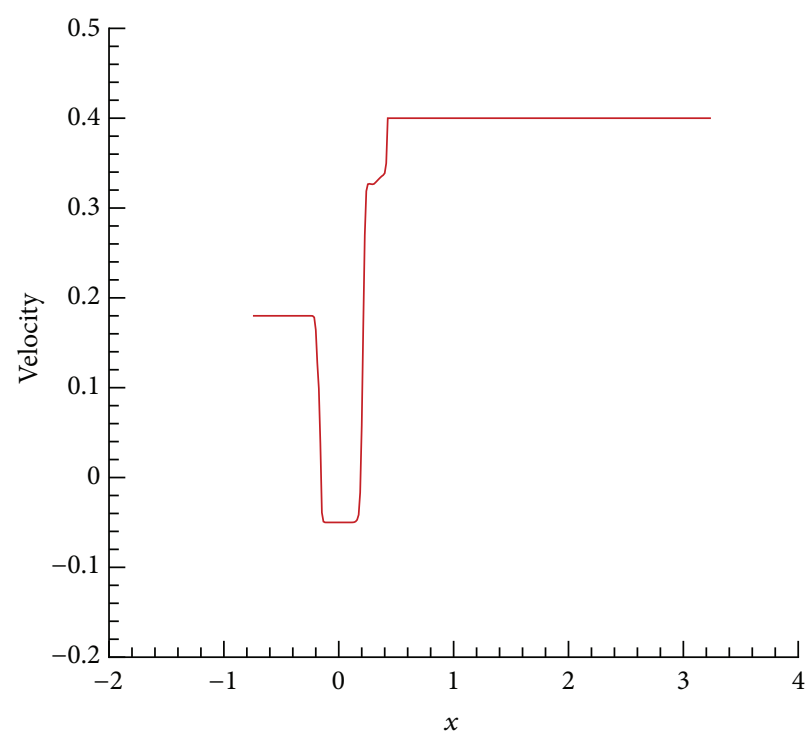

(a)

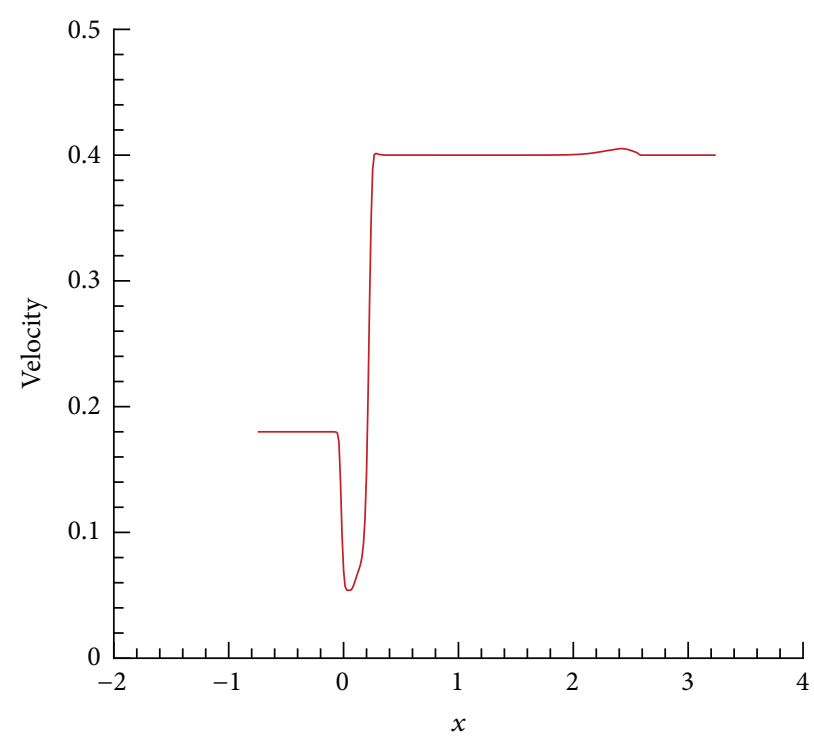

(b)

FIGURE 11: Numerical results of $u$ at $t=0.5$ (a) and $t=5.5$ (b) for Data 3.

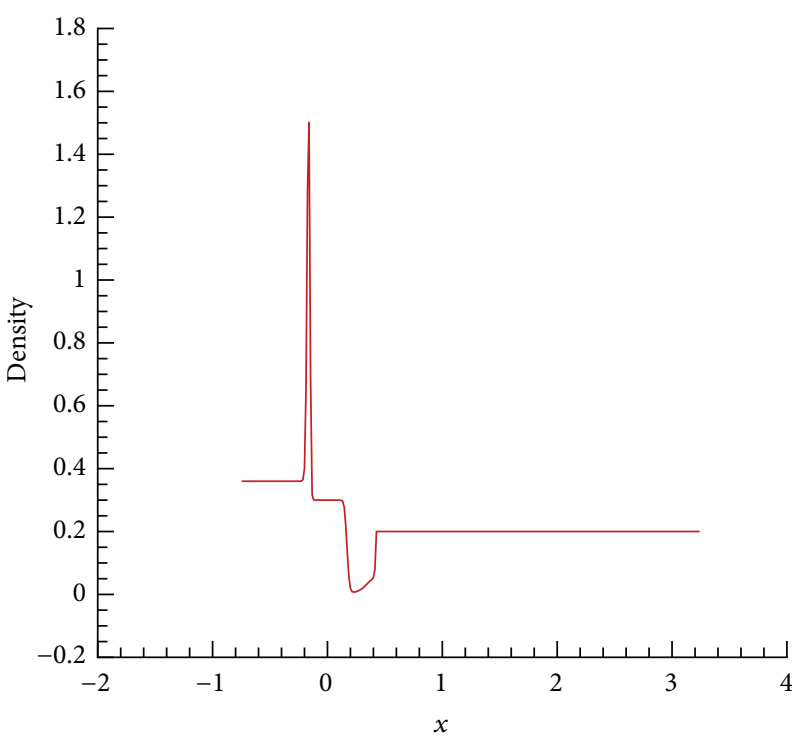

(a)

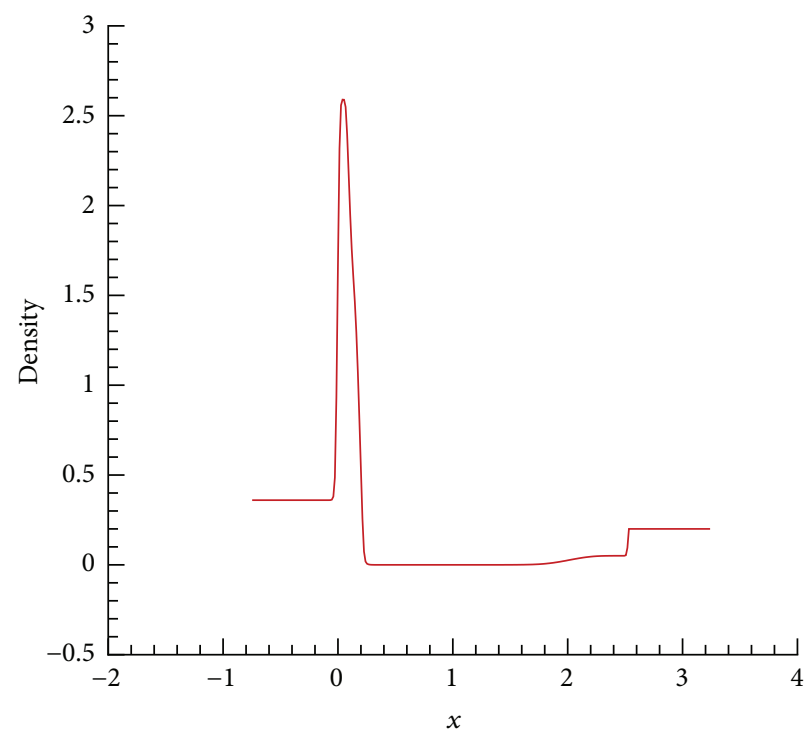

(b)

Figure 12: Numerical results of $\rho$ at $t=0.5$ (a) and $t=5.5$ (b) for Data 3 .

The numerical results are presented by Figures 1416.

From Figures 14-16, we observe that both the contact discontinuities with a vacuum state in between are emitted from $(-0.2,0)$ and $(0.2,0)$ at $t=0.8$, respectively. As time goes on, the vacuum state keeps continuously expanding and never disappears.

To sum up, all of the above numerical results clearly reveal the interactions of delta shock waves and vacuum states discussed in Section 3. We also indicate that because of the occurrence of singularity as the weighted Dirac delta functions, some oscillations appear in the numerical 


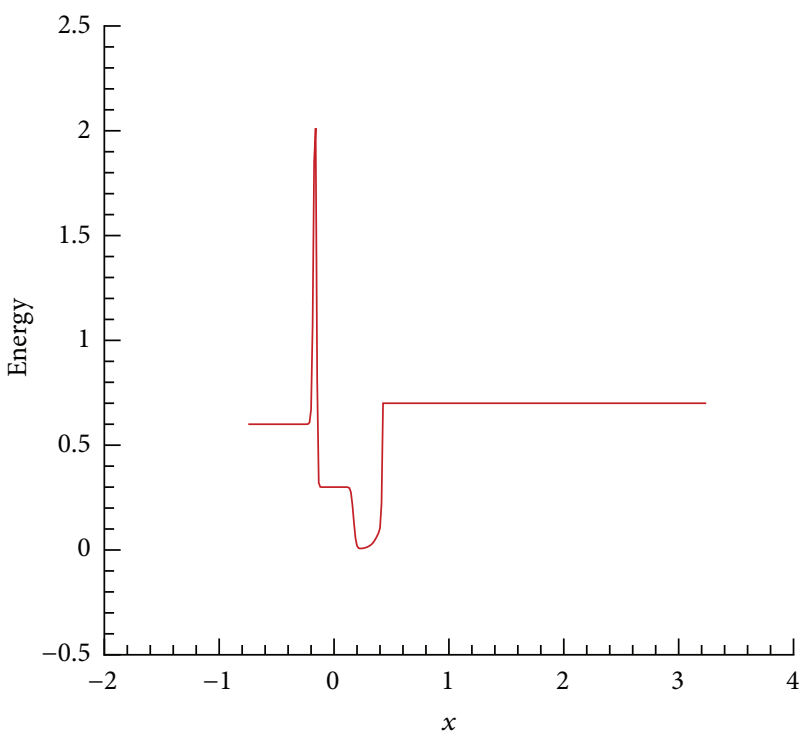

(a)

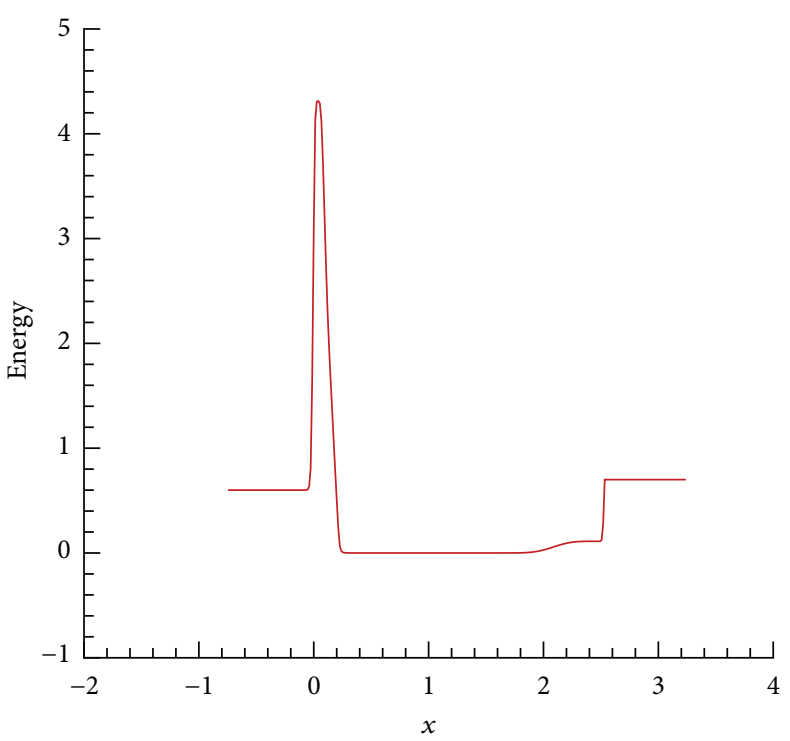

(b)

FIGURE 13: Numerical results of $H$ at $t=0.5$ (a) and $t=5.5$ (b) for Data 3 .

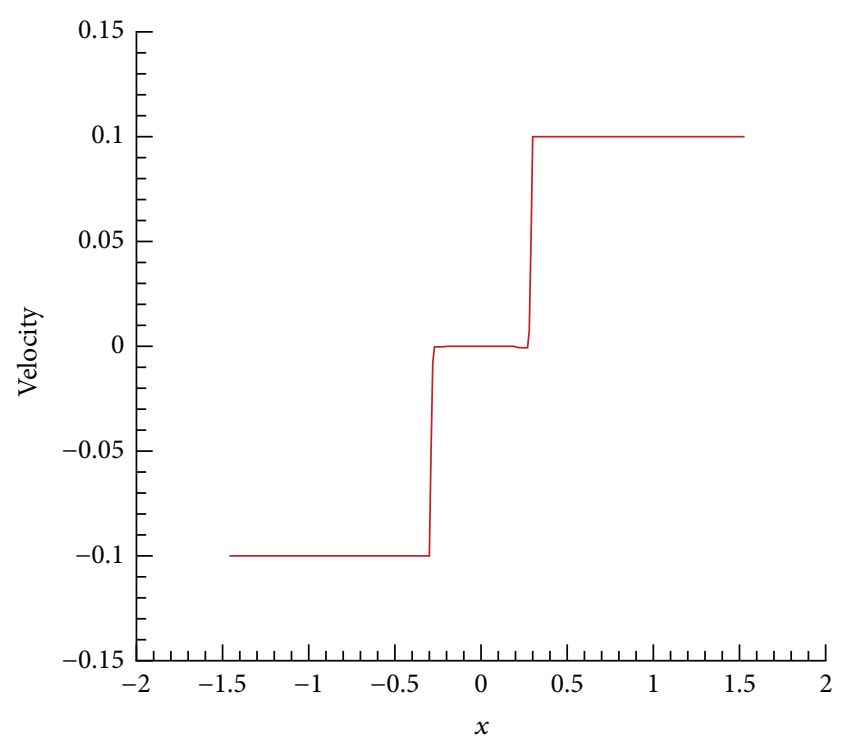

(a)

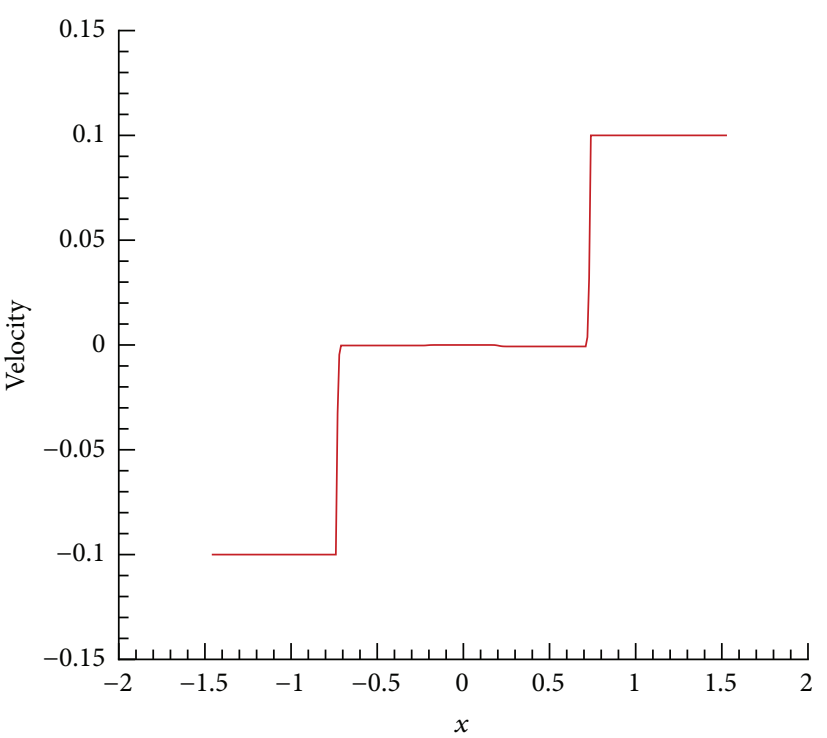

(b)

FIgURE 14: Numerical results of $u$ at $t=0.8$ (a) and $t=5$ (b) for Data 4 .

experiments as shown in Figures 8 and 11. It may be a challenge for numerical schemes when delta shock waves develop in solutions.

\section{Competing Interests}

The authors declare that they have no competing interests.

\section{Acknowledgments}

This work is supported by the National Natural Science Foundation of China (No. 11501488), the Scientific Research Foundation of Xinyang Normal University (No. 0201318), and Nan Hu Young Scholar Supporting Program of XYNU. 


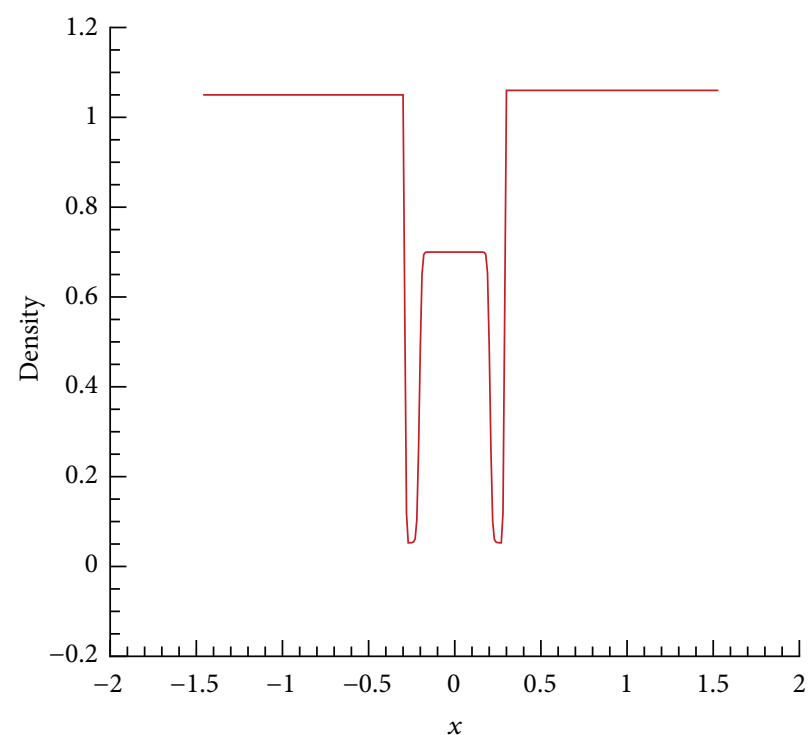

(a)

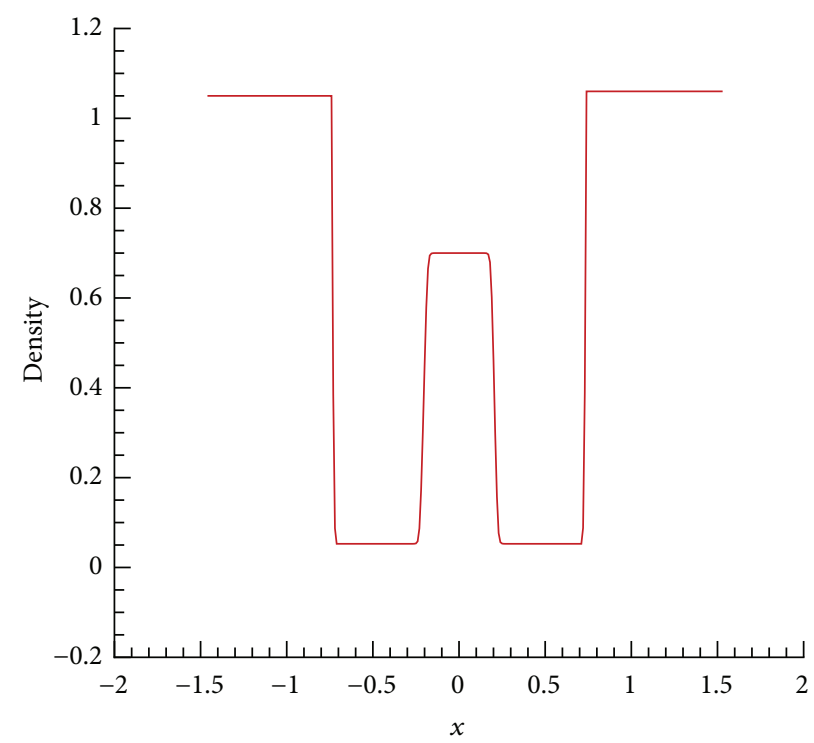

(b)

FIgURE 15: Numerical results of $\rho$ at $t=0.8$ (a) and $t=5$ (b) for Data 4 .

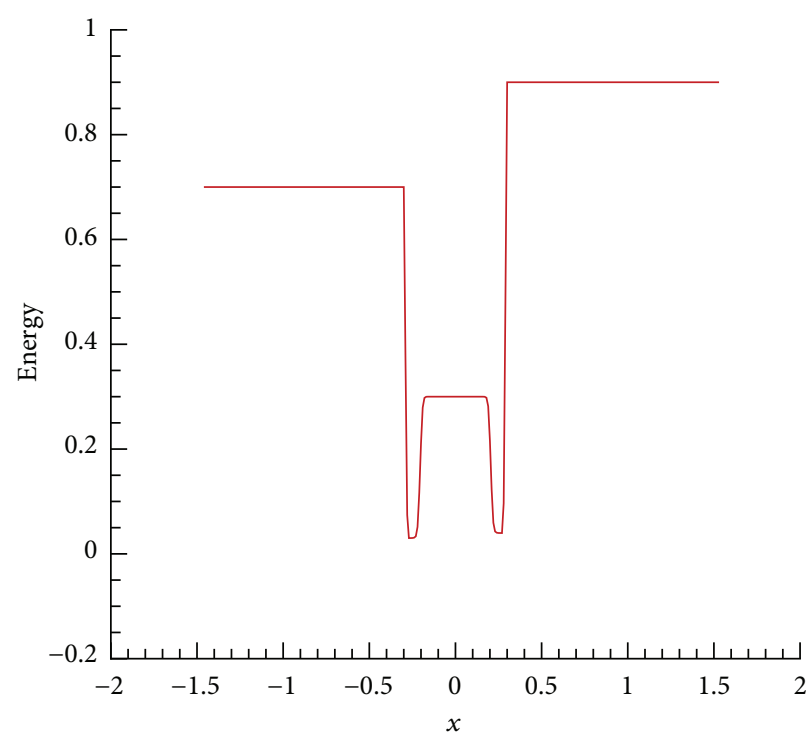

(a)

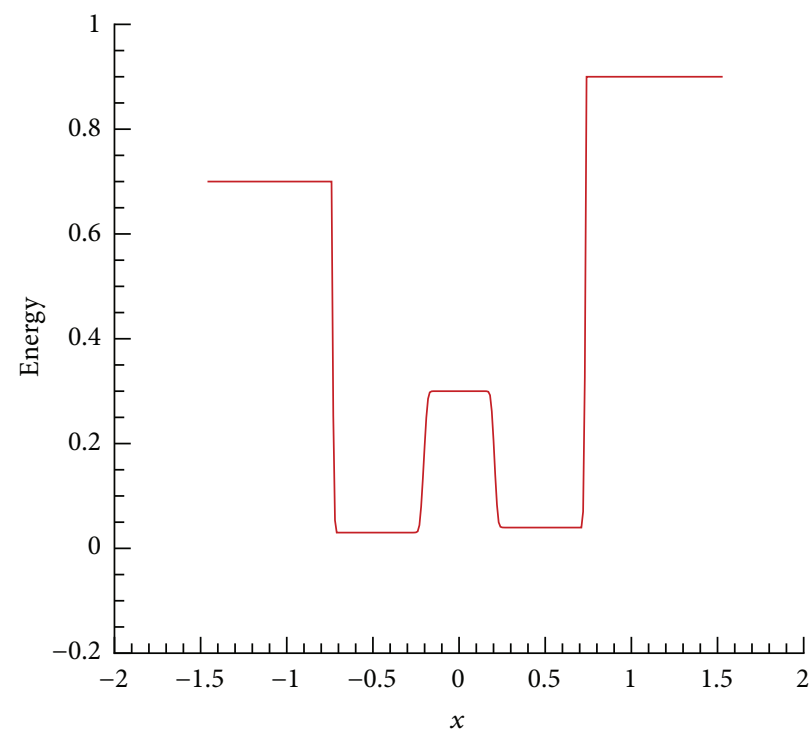

(b)

Figure 16: Numerical results of $H$ at $t=0.8$ (a) and $t=5$ (b) for Data 4.

\section{References}

[1] F. Bouchut, "On zero pressure gas dynamics," in Advances in Kinetic Theory and Computing, vol. 22, pp. 171-190, World Scientific, Singapore, 1994.

[2] R. K. Agarwal and D. W. Halt, "A modified CUSP scheme in wave/particle split form for unstructured grid Euler flows," in Frontiers of Computational Fluid Dynamics, pp. 155-163, World Scientific, Singapore, 1994.

[3] Y. Li and Y. Cao, "Second order large particle difference method," Science China Mathematics A, vol. 22, pp. 171-190, 1985.
[4] Y. Brenier and E. Grenier, "Sticky particles and scalar conservation laws," SIAM Journal on Numerical Analysis, vol. 35, no. 6, pp. 2317-2328, 1998.

[5] S. F. Shandarin and Ya. B. Zeldovich, "The large-scale structure of the universe: turbulence, intermittency, structures in a selfgravitating medium," Reviews of Modern Physics, vol. 61, no. 2, pp. 185-220, 1989.

[6] E. Weinan, Y. G. Rykov, and Y. G. Sinai, “Generalized variational principles, global weak solutions and behavior with random initial data for systems of conservation laws arising in adhesion particle dynamics," Communications in Mathematical Physics, vol. 177, no. 2, pp. 349-380, 1996. 
[7] A. Kraiko, "Discontinuity surfaces in medium without selfpressure," Prikladnaia Matematika i Mekhanika, vol. 43, pp. 539-549, 1979.

[8] B. Nilsson, O. S. Rozanova, and V. M. Shelkovich, "Mass, momentum and energy conservation laws in zero-pressure gas dynamics and $\delta$-shocks: II," Applicable Analysis, vol. 90, no. 5, pp. 831-842, 2011.

[9] B. Nilsson and V. M. Shelkovich, "Mass, momentum and energy conservation laws in zero-pressure gas dynamics and deltashocks," Applicable Analysis, vol. 90, no. 11, pp. 1677-1689, 2011.

[10] S. Albeverio, O. S. Rozanova, and V. M. Shelkovich, "Transport and concentration processes in themultidimensional zeropressure gas dynamics model with the energy conservation law," http://arxiv.org/abs/1101.5815.

[11] D. J. Korchinski, Solution of a Riemann problem for a $2 \times 2$ system of conservation laws possessing no classical weak solution [Ph.D. thesis], Adelphi University, Garden City, NY, USA, 1977.

[12] B. L. Keyfitz and H. C. Kranzer, "A viscosity approximation to a system of conservation laws with no classical Riemann solution," in Nonlinear Hyperbolic Problems, vol. 1402, pp. 185197, Springer, Berlin, Germany, 1990.

[13] H. C. Kranzer and B. L. Keyfitz, "A strictly hyperbolic system of conservation laws admitting singular shocks," in Nonlinear Evolution Equations That Change Type, vol. 27 of The IMA Volumes in Mathematics and Its Applications, pp. 107-125, Springer, New York, NY, USA, 1990.

[14] B. L. Keyfitz and H. C. Kranzer, "Spaces of weighted measures for conservation laws with singular shock solutions," Journal of Differential Equations, vol. 118, no. 2, pp. 420-451, 1995.

[15] P. Le Floch, "An existence and uniqueness result for two nonstrictly hyperbolic systems," in Nonlinear Evolution Equations that Change Type, vol. 27, pp. 126-138, Springer, New York, NY, USA, 1990.

[16] F. Huang and Z. Wang, "Well posedness for pressureless flow," Communications in Mathematical Physics, vol. 222, no. 1, pp. 117146, 2001.

[17] V. Shelkovich, "The Riemann problem admitting $\delta, \delta$-shocks, and vacuum states (the vanishing viscosity approach)," Journal of Differential Equations, vol. 231, no. 2, pp. 459-500, 2006.

[18] W. Sheng and T. Zhang, "The Riemann problem for the transportation equations in gas dynamics," Memoirs of the American Mathematical Society, vol. 137, no. 654, pp. 1-77, 1999.

[19] D. C. Tan and T. Zhang, "Two-dimensional Riemann problem for a hyperbolic system of nonlinear conservation laws. I. Four$J$ cases," Journal of Differential Equations, vol. 111, no. 2, pp. 203254, 1994.

[20] D. C. Tan, T. Zhang, and Y. X. Zheng, "Delta-shock waves as limits of vanishing viscosity for hyperbolic systems of conservation laws," Journal of Differential Equations, vol. 112, no. 1, pp. 1-32, 1994.

[21] H. Yang, "Riemann problems for a class of coupled hyperbolic systems of conservation laws," Journal of Differential Equations, vol. 159, no. 2, pp. 447-484, 1999.

[22] H. Yang and Y. Zhang, "New developments of delta shock waves and its applications in systems of conservation laws," Journal of Differential Equations, vol. 252, no. 11, pp. 5951-5993, 2012.

[23] H. Yang and Y. Zhang, "Delta shock waves with Dirac delta function in both components for systems of conservation laws," Journal of Differential Equations, vol. 257, no. 12, pp. 4369-4402, 2014.

[24] H. Cheng, W. Liu, and H. Yang, "Two-dimensional Riemann problems for zero-pressure gas dynamics with three constant states," Journal of Mathematical Analysis and Applications, vol. 343, no. 1, pp. 127-140, 2008.
[25] H. Yang, "Generalized plane delta-shock waves for $n$ dimensional zero-pressure gas dynamics," Journal of Mathematical Analysis and Applications, vol. 260, no. 1, pp. 18-35, 2001.

[26] V. G. Danilov and V. M. Shelkovich, "Dynamics of propagation and interaction of $\delta$-shock waves in conservation law systems," Journal of Differential Equations, vol. 211, no. 2, pp. 333-381, 2005.

[27] M. Nedeljkov and M. Oberguggenberger, "Interactions of delta shock waves in a strictly hyperbolic system of conservation laws," Journal of Mathematical Analysis and Applications, vol. 344, no. 2, pp. 1143-1157, 2008.

[28] C. Shen and M. Sun, "Interactions of delta shock waves for the transport equations with split delta functions," Journal of Mathematical Analysis and Applications, vol. 351, no. 2, pp. 747$755,2009$.

[29] H. Cheng, "Delta shock waves for a linearly degenerate hyperbolic system of conservation laws of Keyfitz-Kranzer type," Advances in Mathematical Physics, vol. 2013, Article ID 958120, 10 pages, 2013.

[30] H. Cheng, "Riemann problem for one-dimensional system of conservation laws of mass, momentum and energy in zeropressure gas dynamics," Differential Equations \& Applications, vol. 4, no. 4, pp. 653-664, 2012.

[31] G.-S. Jiang and E. Tadmor, "Nonoscillatory central schemes for multidimensional hyperbolic conservation laws," SIAM Journal on Scientific Computing, vol. 19, no. 6, pp. 1892-1917, 1998. 


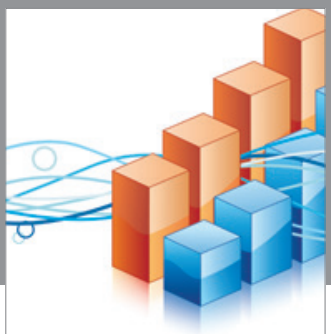

Advances in

Operations Research

vatem alat4

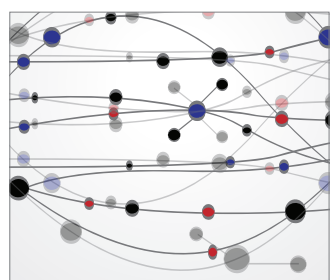

\section{The Scientific} World Journal
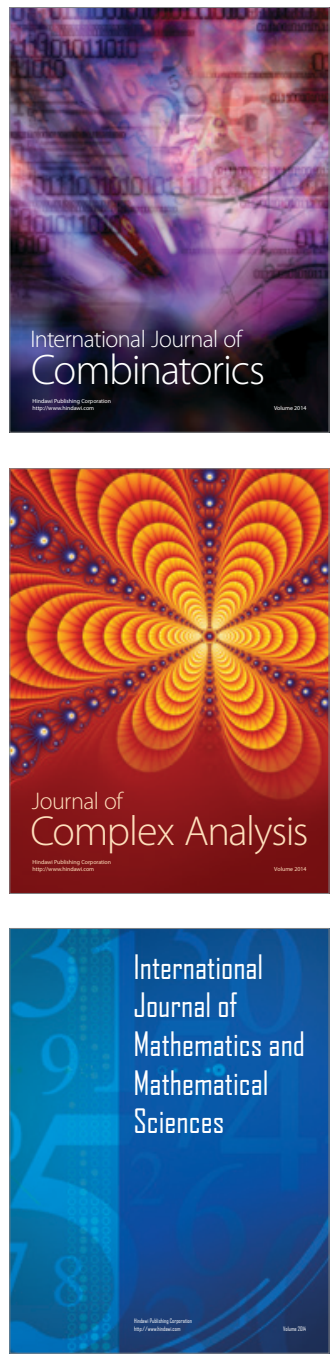
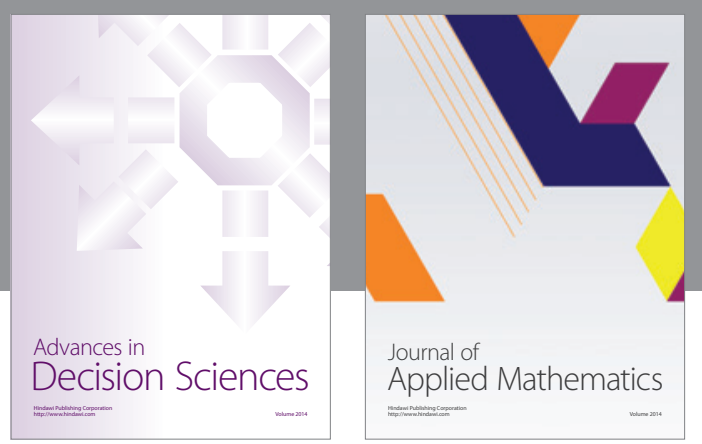

Algebra

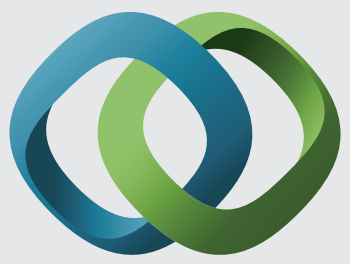

\section{Hindawi}

Submit your manuscripts at

http://www.hindawi.com
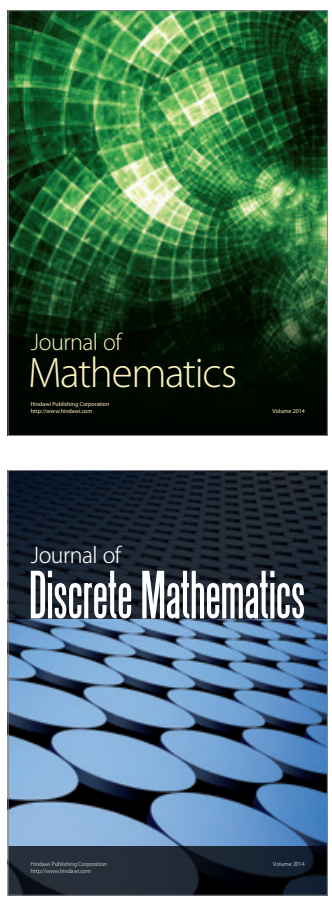

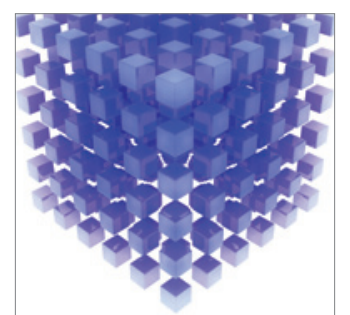

Mathematical Problems in Engineering
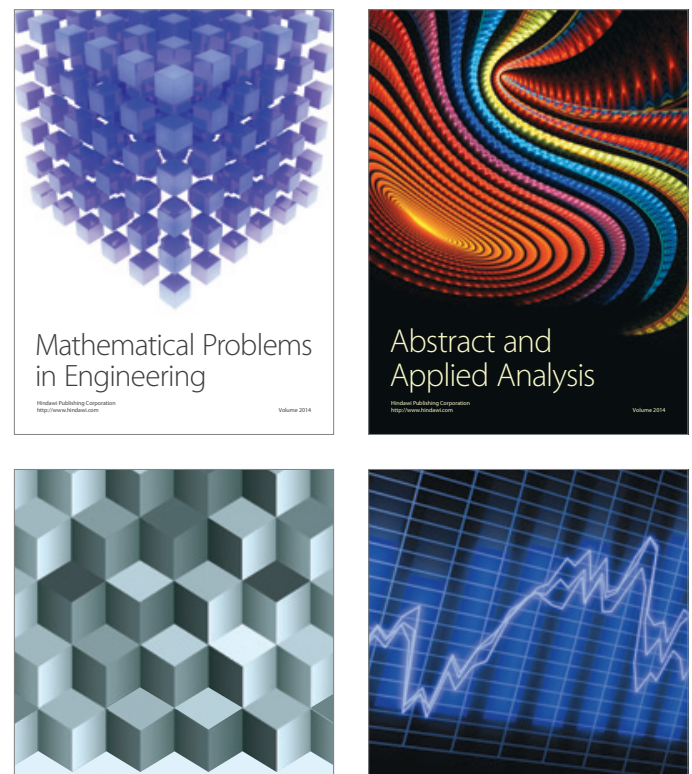

Journal of

Function Spaces

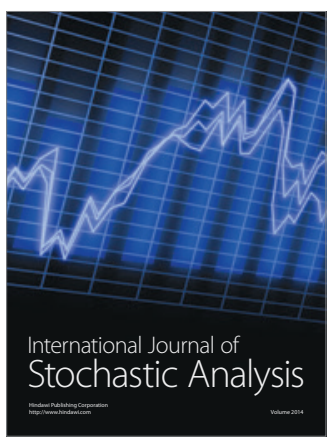

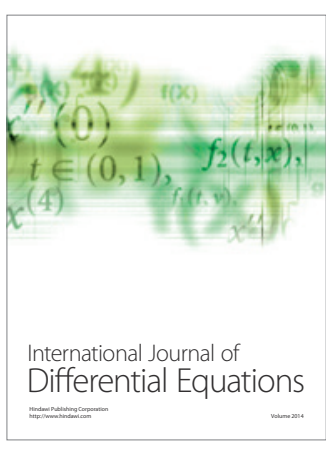
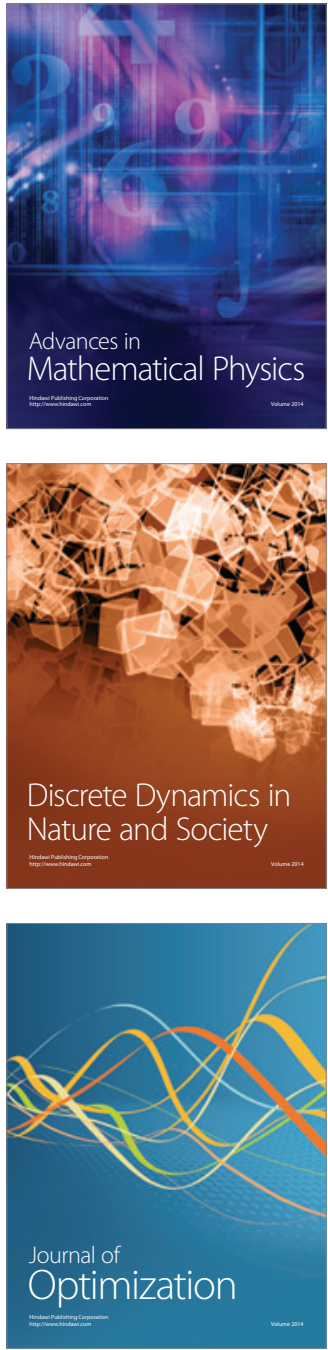